\title{
Zeeman splitting in the diffuse interstellar medium — The Milky Way and beyond
}

\author{
Carl Heiles ${ }^{1}$ and Timothy Robishaw ${ }^{2}$ \\ ${ }^{1}$ Astronomy Department, UC Berkeley \\ email: heiles@astro.berkeley.edu \\ ${ }^{2}$ School of Physics, The University of Sydney \\ email: robishaw@physics.usyd.edu.au
}

\begin{abstract}
We begin with a brief review of Zeeman-splitting fundamentals and the importance of circular polarization, i.e. Stokes $V$. We then turn to modern results in several areas, emphasizing the diffuse interstellar medium in the Galaxy. The median field in the Cold Neutral Medium is determined from $\mathrm{HI}$ absorption lines and is about $6 \mu \mathrm{G}$; the magnetic and turbulent pressures are comparable. Using HI emission lines the field has been mapped in several areas: the field reverses across the Orion Molecular Cloud; the 3-d field structure has been determined in the $\rho$ Oph region; and in regions having shock-like morphology the field is generally stronger, strong enough to limit further compression. We briefly present new field measurements for: photo-dissociation regions at the edges of HII regions, determined from carbon recombination lines; Ultra Luminous Infrared Galaxies, from $\mathrm{OH}$ megamasers; and the 3C 286 damped Lyman- $\alpha$ absorption system, determined from the $21-\mathrm{cm}$ line in absorption. We show the sidelobe response of the Green Bank Telescope, which is surprisingly severe and makes the telescope less than optimum for Zeemansplitting measurements of HI emission lines. Finally, we compare two techniques for determining field strengths, i.e. Zeeman splitting and the Chandrasekhar-Fermi method, and show why the latter usually gives higher field strengths - and sometimes unrealistically high fields.
\end{abstract}

Keywords. ISM: magnetic fields - galaxies: magnetic fields - polarization - techniques: polarimetric

Zeeman splitting probes magnetic fields in many different environments. In this meeting we have heard about its use in probing magnetic fields of stars (Berdyugina, Herpin, Johns-Krull, Reiners, Wade), the X-ray binary Cyg X-1 (Karitskaya), and OH megamasers (Robishaw). Ferland told us about magnetic fields revealed by Zeeman splitting of the 21-cm line in the star-forming regions Orion and M17. The literature on the role of magnetic fields in other star-forming regions is ... not quite vast, but it's quite impressive; for a review, one that includes the physical interpretations of equipartition among turbulent, gravitational, and magnetic energies, see Heiles \& Crutcher (2006).

What hasn't been covered so far in this meeting, nor much in the literature, is the impressive knowledge Zeeman splitting has provided about diffuse regions in the Galaxy. These observations probe the HI line in absorption, which is produced by the Cold Neutral Medium (CNM), and in emission, which is produced by HI gas of all temperatures. We will also mention very briefly some other new, unpublished $3 \sigma$ results in dense regions (photodissociation regions-PDRs) and also extragalactic magnetic fields seen in Zeeman splitting of $\mathrm{OH}$ megamasers and damped Lyman- $\alpha$ (DLA) absorption systems. We begin, however, by briefly reviewing some fundamentals; these are intimately familiar to those working in the Zeeman business, but not so to many who study magnetic fields using other techniques. 


\section{Zeeman splitting fundamentals and Stokes parameters}

When a simple atom like hydrogen (HI) sits in a magnetic field, the lines suffer Zeeman splitting. Here we shall focus on the $21-\mathrm{cm}$ line, which radio astronomers use to study the diffuse interstellar medium. In a magnetic field of $1 \mu \mathrm{G}$, the line splits into two components centered at different frequencies; the separation is $2.8 \mathrm{~Hz}$, i.e. $\pm 1.4 \mathrm{~Hz}$ from line center. These so-called $\sigma$ components are polarized. If the field lies along the line of sight, then they are $100 \%$ circularly polarized, with opposite senses for the two frequencies. Classically, this corresponds to the electrons gyrating around the field in the clockwise and counterclockwise directions. Now turn the field so it is perpendicular to the line of sight; then the gyrating electrons appear to oscillate in straight lines across the field lines, producing linear polarization oriented perpendicular to the field. So the polarization of the $\sigma$ components changes from pure circular to pure linear as the field changes from line-of-sight to plane-of-sky. Finally, electrons can oscillate in straight lines oriented along the field lines, and then the field has no effect on the frequency. The so-called $\pi$ component has no frequency shift and is linearly polarized parallel to the plane-of-sky field; the intensity goes to zero when the field becomes line-of-sight, because then the classical electrons are oscillating to and from along the line of sight.

First consider the Stokes parameter $V$, which is the difference between the two circular polarizations, for the case when the ratio of Zeeman splitting to line width - call this ratio $R$-is small. For a line-of-sight field, Stokes $V$ sees the intensity difference between two $\sigma$ components that are centered at different frequencies. So the Stokes- $V$ shape is the frequency derivative of the line, and the amplitude is proportional to $R \cos \theta$, where $\theta$ is the angle of the field to the line of sight. The $\cos \theta$ arises because the circular polarization intensity $\propto \cos \theta$. When $R$ is small, Stokes $V$ is small; Zeeman observations are usually sensitivity-limited and we need lots of observing time.

Now consider the linear-polarization Stokes parameters $Q$ and $U$, for which the sensitivity situation is much worse: the difference between linearly polarized intensities for position angles parallel and perpendicular to the plane-of-the-sky field is a difference in line width, which means that the shape looks like the second derivative of the line with an amplitude $\propto R^{2} \sin ^{2} \theta$. With $R \ll 1$, this makes the intensity of linear polarization impossibly weak. The watchword for Zeeman-splitting specialists is that, for weak lines having $R \ll 1$, circular polarization is the only way to go.

Radio astronomers use cross-correlation techniques to measure all four Stokes parameters simultaneously (Heiles 2002, Heiles et al. 2001). With native linear polarization from the feeds (call them $X$ and $Y$ ), Stokes $U$ is the $X Y$ product and Stokes $V$ is the $X Y$ product with a $90^{\circ}$ phase shift. Cross-Correlation is an exquisitely sensitive and robust technique, so this situation is highly desirable compared to using feeds with native circular polarization (call them $R$ and $L$ ). In this latter case, Stokes $V$ is the difference $R^{2}-L^{2}$; this difference between two large numbers is subject to small gain variations in the electronics. For measuring Zeeman splitting with the $R \ll 1$ case, we have the seemingly paradoxical situation that native linear feeds are better.

\section{Zeeman splitting in $\mathrm{HI}$ absorption: history and current status}

Zeeman splitting of the 21-cm HI line was discovered by Verschuur (1969) by looking at the line in absorption against two strong radio sources, Cas A and Tau A. Amazingly enough, no further work was done in HI absorption for 32 years - not for lack of suitable equipment (Arecibo would have been superb, even before its final upgrade in the late 1990s) but more because (1) it's not easy to measure weak polarization (it takes lots of 
time and careful attention to detail) and (2) the few observers willing to spend the effort concentrated on mapping the field in HI emission. We cover emission in the next section.

Finally, just before the new millennium, Troland and Heiles - over some glasses of Jack Daniel's whiskey - conceived the Millennium Survey of Zeeman splitting in HI absorption of all feasible sources in the Arecibo sky. They observed 79 sources (different directions in the sky) and examined 69 Gaussian components for which the derived fields and errors were well-defined. This is the largest statistical body of magnetic field information on the diffuse ISM. A big bonus is that the Gaussian components give line widths, kinetic temperature, and column density. Heiles \& Troland (2005) discuss the scientific implications of the statistics. The principal conclusions are that the median CNM magnetic field is about $6 \mu \mathrm{G}$, the typical temperature $50 \mathrm{~K}$, the median $\mathrm{HI}$ column density for $|b|>10^{\circ}$ is $\sim 0.5 \times 10^{20} \mathrm{~cm}^{-2}$ (far smaller than the generally accepted "standard Spitzer cloud," which is about $3 \times 10^{20} \mathrm{~cm}^{-2}$ ), and - as seems to be usual in astrophysical situations - the turbulent and magnetic energy densities are comparable, both much larger than the gas thermal energy density (i.e., it's a low- $\beta$ plasma).

\section{Zeeman splitting in $\mathrm{HI}$ emission: history and current status}

The field of ISM Zeeman observing wasn't dead during thosee 32 years between absorption line studies. Heiles and Troland used the Hat Creek 85-ft, and Verschuur used the NRAO 140-footer, to look at $\mathrm{HI}$ in emission in the hope of making magnetic field maps. Others used Arecibo, Westerbork, the 140-foot, and the VLA to measure Zeeman splitting, mostly in molecular clouds and $\mathrm{OH}$ masers. Verschuur's work was limited by the time one can hope to get from a national-observatory multipurpose telescope. Heiles and Troland had the Hat Creek 85-ft telescope almost to themselves and were able to make real progress in dealing with sensitivity and instrumental concerns (Troland \& Heiles 1982; Heiles 1989). And technically, the 85-foot's electronics were fully state-of-the-art, particularly with the advent of a 1024-channel digital correlator after Troland earned his Ph.D. This led to a series of 5 papers (Heiles 1988, Heiles 1989, Goodman \& Heiles 1994, Myers et al. 1995, Heiles 1997) in which a total of 429 positions had sufficiently reliable field strengths to publish. Some of these yielded maps of large regions: in Orion, where a large-scale reversal was seen across the Orion Molecular Cloud (Heiles 1997); in Ophiuchus, where the stellar polarization and HI Zeeman splitting offered a 3-dimensional characterization of the field morphology (Goodman \& Heiles 1994); and the North Celestial Pole (Myers et al. 1995), where there is a 25-degree diameter expanding shell with enhanced field $(\sim 10 \mu \mathrm{G})$ in the compressed shell.

Figure 1 projects the totality of 429 positions onto an image of the integrated HI line. The coverage is sparse in most regions, and for weak fields the results are somewhat uncertain because of instrumental polarized sidelobes (mainly beam squint; see below), but they offer a robust conclusion: the observed fields are high in regions where the HI morphology suggests we see an edge-on shock. This, then, is consistent with our expectation of field amplification in dense gas because of flux freezing. Moreover, the field is strong and limits the gas compression.

These measurements are regarded with considerable suspicion by many astronomers, especially European astronomers, because of Verschuur's Indictment. In a pair of papers (Verschuur 1995a, 1995b), amplified by a series of colloquia given mainly to European astronomers in the mid 1990s, Verschuur claimed that every single Zeeman-splitting detection in HI emission was bogus because of polarized sidelobes interacting with the HI velocity field. Most serious is "beam squint," in which the two circular polarizations point in slightly different directions on the sky. For most telescopes [the Hat Creek 


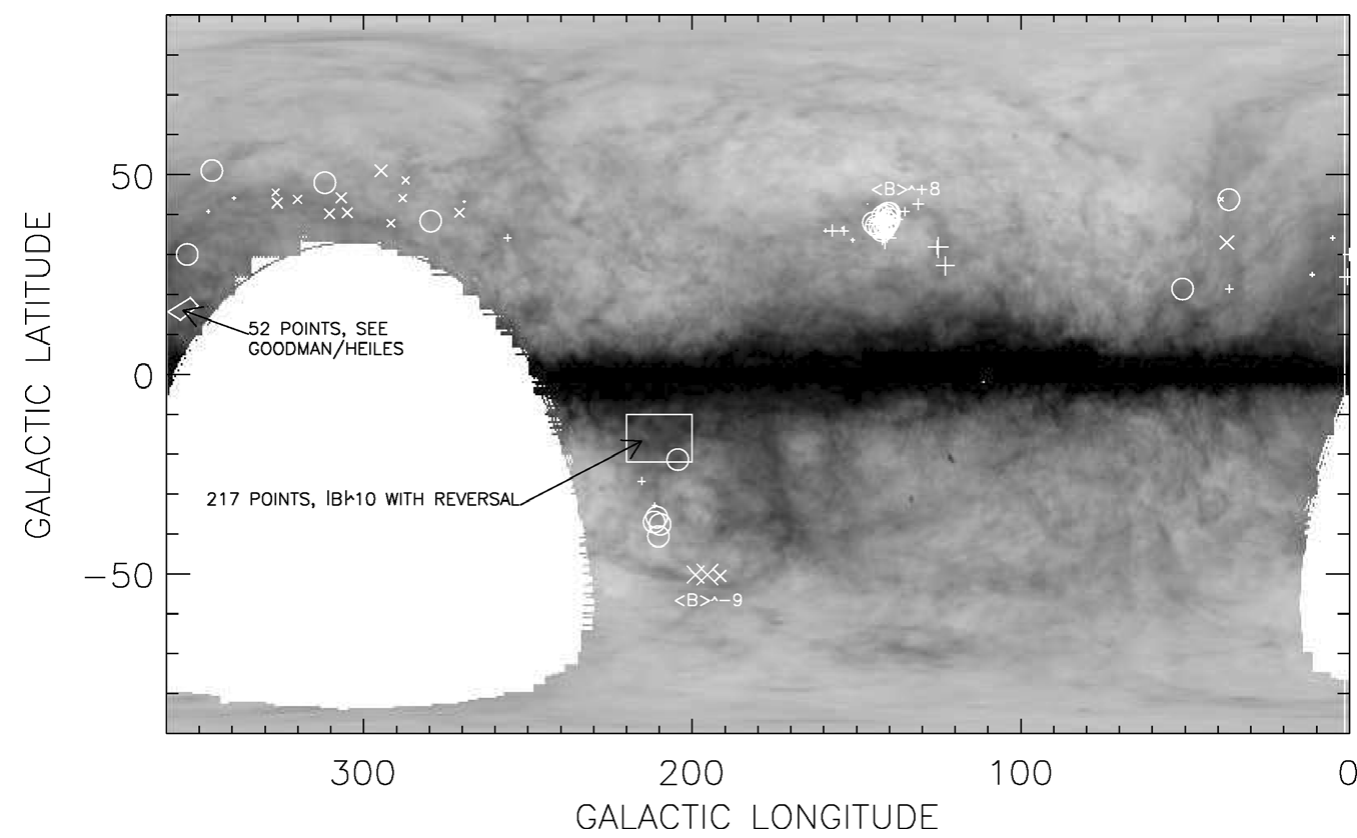

Figure 1. The 429 positions at which the 5 Hat Creek papers reported magnetic fields derived from HI in emission.

85 foot (Heiles 1996), the Arecibo telescope (Heiles 1999), the Green Bank Telescope (Robishaw \& Heiles 2009)], this position difference is $\sim 1$ arcsec. For the 140 foot, the squint changes with time, probably as a result of feed-mounting details, and is usually bigger: $\sim 7$ arcsec (Verschuur 1969); unmeasurably small (Verschuur 1989); and $\sim 2$ arcsec (Verschuur 1995a). A 1-arcsec beam squint doesn't seem like much, but it can be serious: a velocity gradient of $1 \mathrm{~km} \mathrm{~s}^{-1} \mathrm{deg}^{-1}$ that is aligned with the direction of beam squint produces a frequency difference of $5 \mathrm{~Hz}$ between the two circular polarizations, which is equivalent to $1.6 \mu \mathrm{G}$. Verschuur claimed that all results were simply fake, caused by beam squint. This, however, is simply not the case. Most telling, many GBT results match the Hat Creek ones - in particular, at the well-studied North Celestial Pole.

Heiles (1996) thoroughly studied the response of the Hat Creek telescope to Zeeman splitting at the NCP and provides a detailed measurement of the uncertainties in the derived field for that position, for which the HI velocity gradient is somewhat larger than usual. Finally, Heiles (1998), in an unfamiliar and rarely-quoted journal, thoroughly discusses Verschuur's criticisms and explains why they are exaggerated. The bottom line: most of the emission results are reasonably accurate.

\section{The Green Bank Telescope as a case study in polarized sidelobes}

Beam squint isn't all there is, as Robishaw and Heiles (2009) discovered for the GBTmuch to our dismay. We pointed the GBT at the NCP, staring at it for a full 24 hours. There were significant variations in both Stokes $I$ and $V$ with time. For a telescope with small beam squint, and also a clear aperture so that there should be no other sidelobes, this was not only a disappointment but also raised a puzzling question: Why? How did these time variations arise?

They arise from spillover of the feed's illumination of the secondary mirror. This produces three different sidelobe components: $(1)$ a $\sim 40^{\circ}$-diameter doughnut-like pattern 


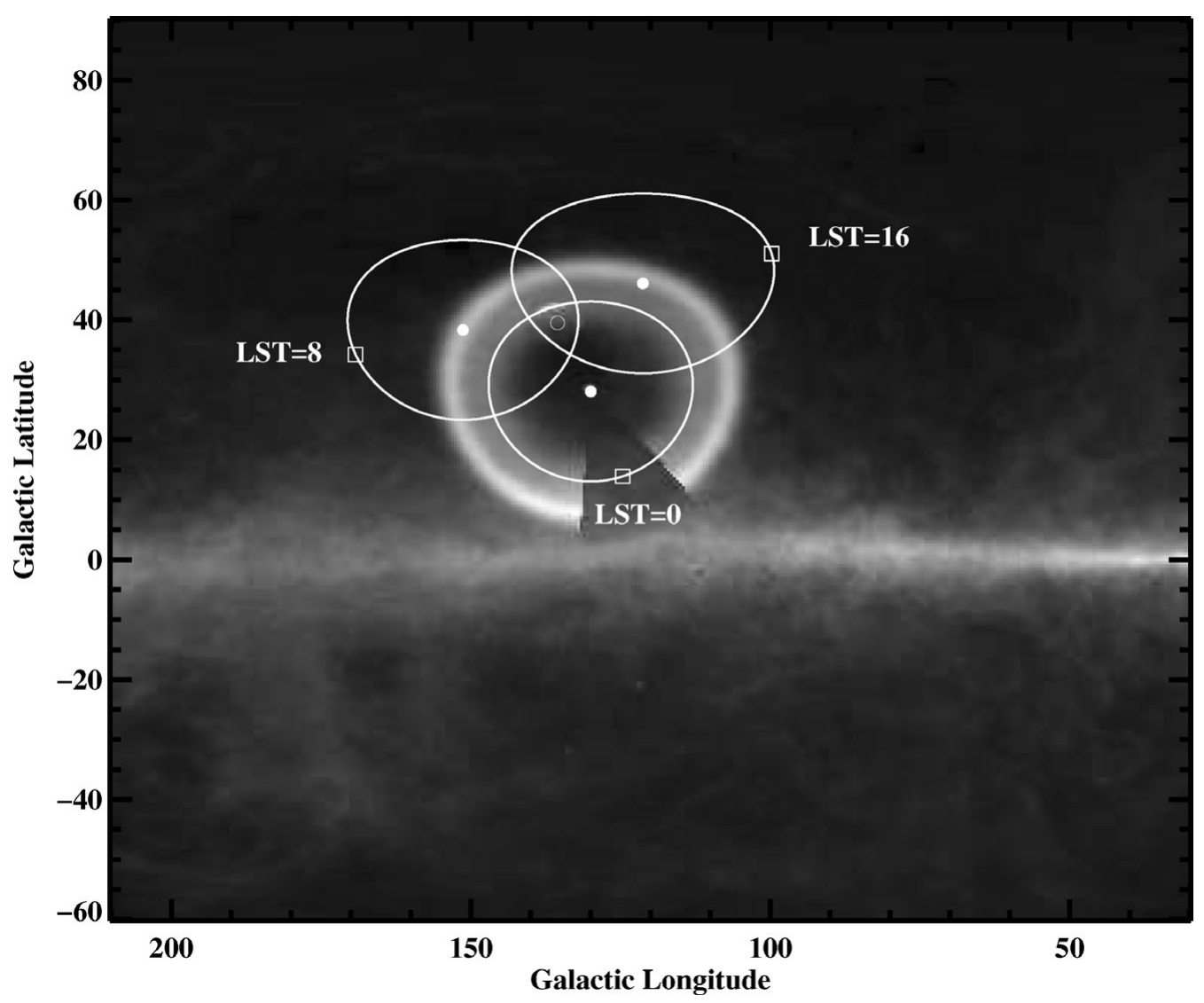

Figure 2. Images of the secondary-reflector sidelobes at three LSTs for the position G135.5+39.5, superposed on the integrated $21-\mathrm{cm}$ line intensity from the LAB survey. The empty circle is the source position. The filled circles are the Arago spot. The solid $30^{\circ}$-diameter circles approximately show the subreflector edge as seen by the feed. Each of the three squares lies on its subreflector circle just inside the feed arm. The circular greyscale image has whiteness proportional to the spillover pattern; this is shown only for LST $=0$. The blanked-out portion of the spillover pattern is the angular slice of the screen.

centered on the line joining the centers of the feed and the secondary mirror; (2) the "Arago spot," which is the diffraction spike at the center of an occulting disk; and (3) a "screen" component associated with the spillover radiation interacting with the feed leg, which has a protective screen intended to control this aspect of the spillover radiation. Components (1) and (3), especially (1), tend to be the most serious for Stokes I; component (3) is most serious for Stokes $V$. Component (1) is well-enough known so that its Stokes- $I$ contribution is quite accurately predictable. Unfortunately, the same is not true for component (3), especially in Stokes $V$.

Figure 2 depicts these feed/secondary sidelobe components on the sky, superposed on an image of the total HI line intensity. As the sky turns, component (1) sweeps around the source, covering a total sky area that is bounded by a small circle of radius $\sim 40^{\circ}$. The other components move around the observed position as the parallactic angle changes. This polarized-sidelobe contribution to Stokes $V$ cannot be accurately predicted, which makes the GBT a less desirable telescope than we would have hoped for measuring Zeeman-splitting of $\mathrm{HI}$ in emission. 


\section{Three new results in $\mathrm{HI}$ emission}

One: the NCP shell. Above we mentioned the high field strengths in the NCP shell. This shell has fascinating structure on the $\sim 3$ arcmin scale, as revealed by the $100 \mu \mathrm{m}$ IRAS emission; moreover, the measured field strengths from Zeeman splitting are high, easily reaching $10 \mu \mathrm{G}$ - but this is with 36 -arcmin resolution, which is much worse than the typical structural scale. A few positions measured with the Effelsberg telescope show higher fields (Myers et al. 1995). We have used the GBT to map a very large region containing the NCP shell. These mapping data also include Stokes $V$; while we won't have the sensitivity to map the field with the full GBT resolution, by averaging in area we will obtain a large-scale map of the field strength, albeit limited in angular resolution. Currently we have mapped only the Stokes $I$, which is itself intriguing. Figure 3 shows this map at one particular velocity, $2.6 \mathrm{~km} \mathrm{~s}^{-1}$, together with Zeeman-derived magnetic field measurements for a few individual positions. Unfortunately, we don't have space to include other velocities. The structure changes markedly with velocity - a circumstance that indicates that the structure is not filamentary, but rather sheetlike: what seems like filaments is simply the cusps where the sheet lies tangential to the line of sight.
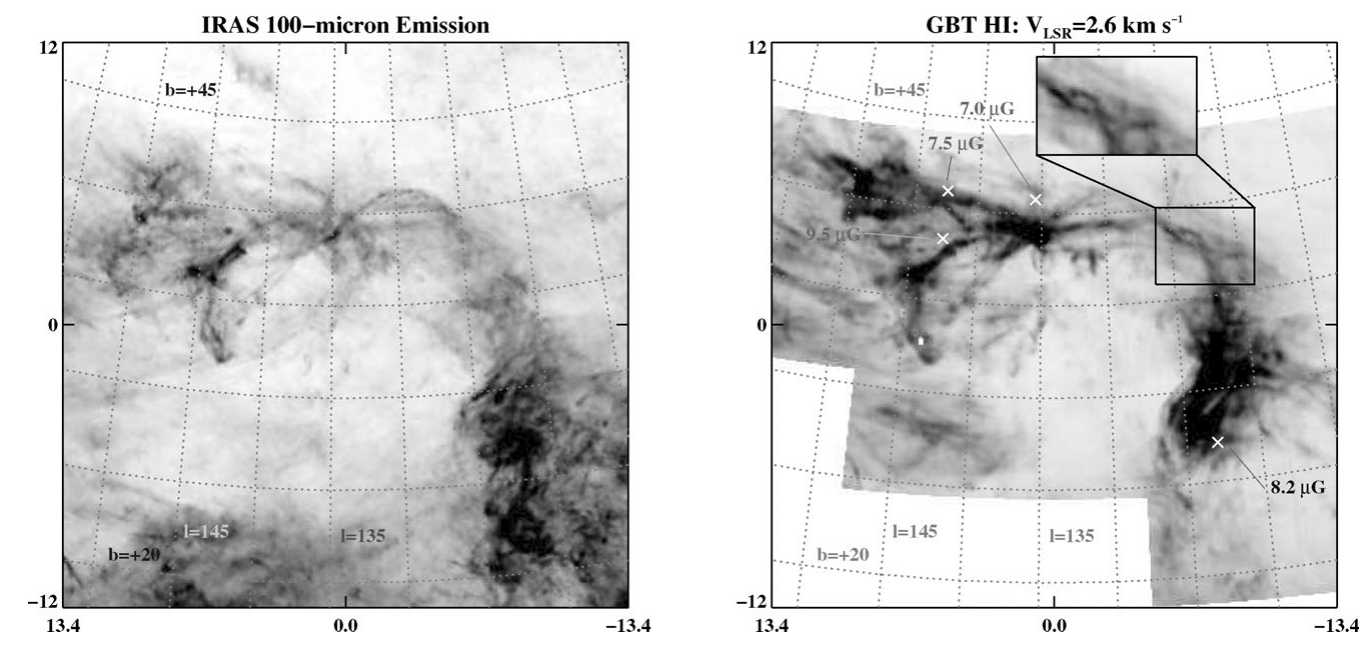

Figure 3. Left, the $100 \mu \mathrm{m}$ IRAS image of the North Celestial Pole (NCP) loop; right, the HI image for $V_{\mathrm{LSR}}=2.6 \mathrm{~km} \mathrm{~s}^{-1}$. The numbers on the right panel are $B_{\|}$, which is strong. All images are negatives, in which black means higher intensity. The graticule spacing is $5^{\circ}$.

Two: The Orion Molecular Cloud. Above we mentioned the Hat Creek result for the Orion Molecular Cloud, which may indicate that a helical field envelopes the cloud. Our GBT results amplify this possibility, because they show that the reversal is very sharp: it's limited by the angular resolution of the GBT.

Three: Enhanced Faraday Rotation Spots near the Taurus Molecular Cloud. Wolleben \& Reich (2004) made a beautiful map of Faraday rotation measure (RM) for an area around the Taurus Molecular Cloud (TMC) and found nine well-defined spots having small RM. They used $\mathrm{H} \alpha$ data to place an upper limit on the electron density, which provided a lower limit on the line-of-sight field $B_{\|} \gtrsim 20 \mu \mathrm{G}$. They suggested that these spots are associated with the TMC, possibly associated with recently-formed stars. We obtained 21-cm line Zeeman-splitting data for 3 of these positions and do not find such strong fields for velocities near to the TMC velocity. However, we do find fields of $\sim 50 \mu \mathrm{G}$ for a $-50 \mathrm{~km} \mathrm{~s}^{-1}$ component. We emphasize that these derived fields have not been corrected 
for the GBT sidelobes, so these detections may not be real. Nevertheless, it is interesting to take these measurements at face value - i.e., as being real - and to superpose the 9 RMminimum positions on a grey scale map of the $-50 \mathrm{~km} \mathrm{~s}^{-1}$ component. Figure 4 shows these spots projected against both the TMC and this component. Morphologically, the case for their association with the $-50 \mathrm{~km} \mathrm{~s}^{-1}$ component is at least as good as with the TMC. If so, the shock could have produced the enhanced field and, also, an enhanced electron density.
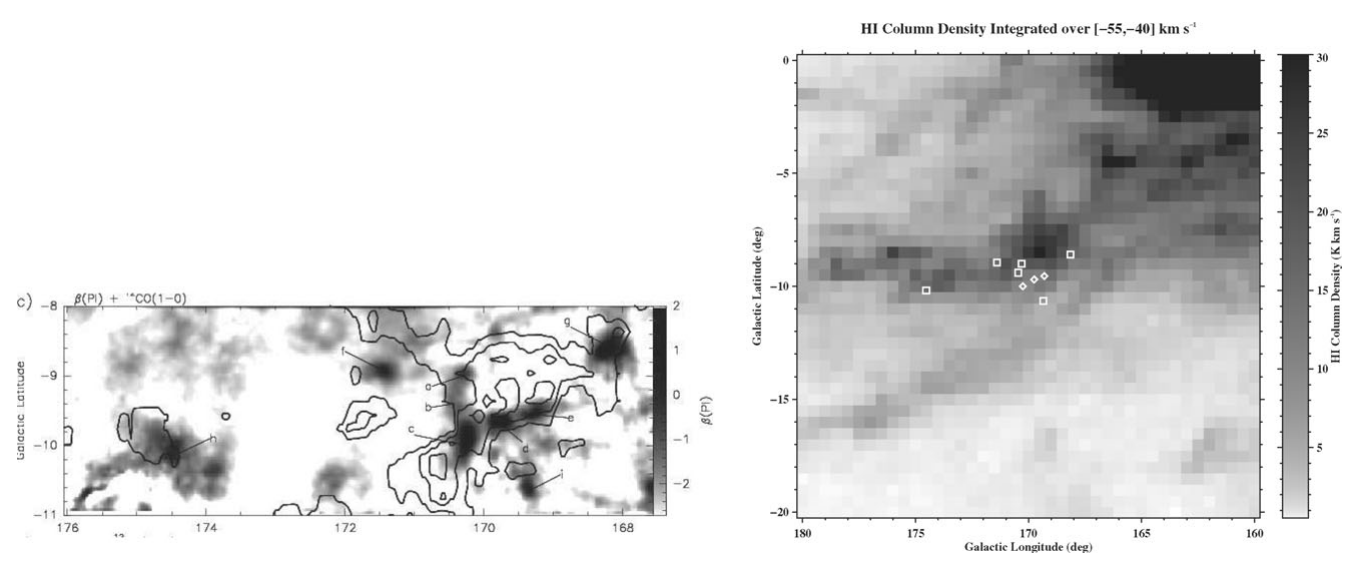

Figure 4. Left, in greyscale is the Wolleben \& Reich (2004) RM map and the contours show the boundary of the Taurus Molecular Cloud. The 9 RM minima (dark spots) are labelled. Right, the $-50 \mathrm{~km} \mathrm{~s}^{-1} \mathrm{HI}$ emission intensity (area of the Gaussian) versus position, with the same 9 minimum-RM spots.

\section{New results for small sources}

Zeeman splitting measurements of unresolved sources suffer no contamination from polarized sidelobes, so one is limited only by signal-to-noise. Here we mention three recent results.

One: Magnetic Fields in PDRs. The interface between an HII region and its neutral surroundings is bounded on the outside by a shock and on the inside by an ionization front. The pressure is comparable to that in the HII region. Within the PDR, the ambient gas has been compressed by the shock and the density has increased by roughly the ratio of the temperatures in the HII region and the ambient medium - a factor $\sim 100$. In a one-dimensional shock, the field strength should increase by the same factor, so the field strength in PDRs should be in the $\mathrm{mG}$ range. We used the GBT to observe 8 CRRLs simultaneously to measure Zeeman splitting. We examined 5 sources and obtained $3 \sigma$ detections for two: DR21 $(-916 \pm 305 \mu \mathrm{G})$ and M17 $(2530 \pm 864 \mu \mathrm{G})$. In the Zeeman-splitting game one is not comfortable with just $3 \sigma$ detections, but these are very suggestive. In particular, these sources are small and should have small angular structure in the density and magnetic field structure. Rather than pursue higher sensitivity with a single dish like the GBT, we feel the current results justify a significant time commitment with the VLA.

Two: $O H$ Megamasers. It is surprisingly easy to detect Zeeman splitting in $\mathrm{OH}$ megamasers. This offers a unique probe into the magnetic properties of the host galaxy, which is always an Ultra Luminous IR Galaxy (ULIRG), usually at moderate redshift. We are 
pursuing an active program to observe these galaxies; Robishaw (this meeting) discusses Zeeman splitting in OHMs.

Three: DLAs. We have looked for Zeeman splitting in four DLA systems against 3 sources, with the results given in Table 1 . The first entry has an impressively small upper limit and the next two have less impressive upper limits. What's really impressive is the fourth entry for $3 \mathrm{C} 286$, an $84 \mu \mathrm{G}$ field for a $z=0.692 \mathrm{DLA}$. Figure 5 shows this result.

Table 1. Zeeman-Splitting Observations of DLAs

\begin{tabular}{crrr}
\hline System & $\frac{N(H I)}{10^{20}}$ & $B_{\|}, \mu \mathrm{G}$ & Ref \\
\hline $0738+313, z=0.0912$ & 15 & $1.8 \pm 2.4$ & 1,2 \\
$0738+313, z=0.2212$ & 8 & $12.5 \pm 9.0$ & 1,2 \\
$0235+164, z=0.524$ & 30 & $\lesssim 10$ & 3 \\
3C 286, $z=0.692$ & 30 & $84 \pm 9.0$ & 4
\end{tabular}

Ref 1: Turnshek et al. (2001) (DLA properties)

Ref 2: Lane \& Heiles (2008) (Zeeman splitting)

Ref 3: Wolfe et al. (1982) (DLA properties)

Ref 4: Wolfe et al. (2008) (Zeeman splitting)

Note: Field strengths are unpublished except for Ref 4 .
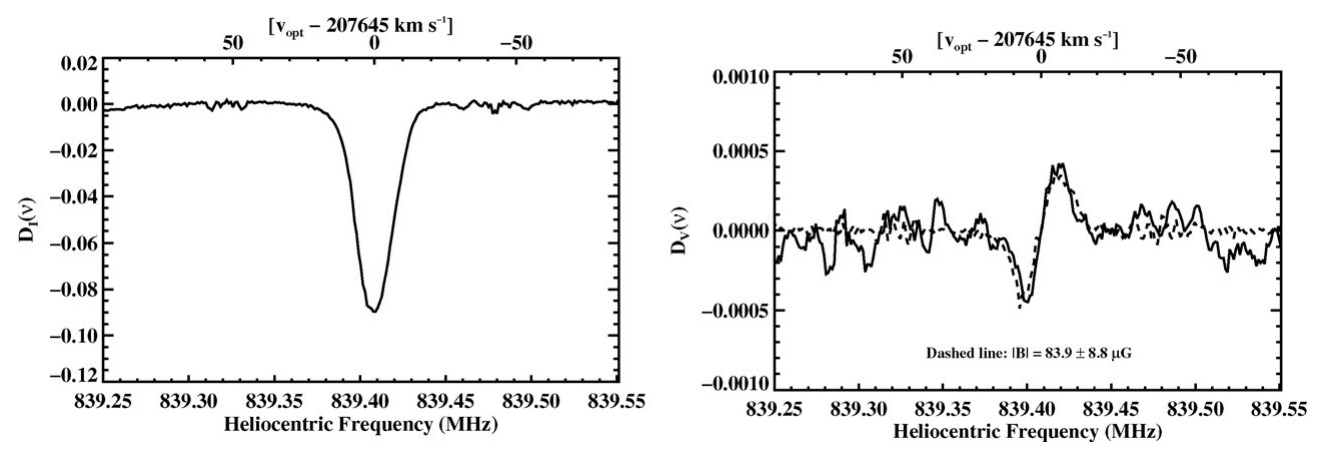

Figure 5. The 3C 286 DLA in the 21-cm line. Left, Stokes $I$; right, Stokes $V$. On the right, the solid line is the data and the dashed line is the best fit.

This field is exceptionally high: the magnetic pressure $\sim 10^{8} \mathrm{~cm}^{-3} \mathrm{~K}$, more than $10^{4}$ higher than the anticipated CNM thermal pressure. But there are additional amazing oddities about this DLA: (1) its column density $N(H I) \sim 3 \times 10^{21} \mathrm{~cm}^{-2}$, about 100 times bigger than Galactic CNM clouds; (2) VLBI studies (Wolfe et al. 1976) show that the DLA cloud is at least 200 pc in size, about 100 times bigger than Galactic CNM clouds; (3) its velocity dispersion $\sim 3.8 \mathrm{~km} \mathrm{~s}^{-1}$, so the velocity gradient across the cloud $\lesssim 0.02 \mathrm{~km} \mathrm{~s}^{-1} \mathrm{pc}$; this is tiny, especially in view of the huge magnetic pressure!

This cloud is very weird, even without the strong field. The strong field makes it even weirder, so one might wish to discount the observation as being bogus. This is possible, and the result needs confirmation, but it does seem real for the following reasons: (1) the result repeats from day to day for our 8-day series of observations; (2) the result is statistically robust, with the average and median giving comparable results; and (3) most telling, Stokes $U$ has no signal. This is important: Stokes $V$ and Stokes $U$ are both derived from cross-correlations of native linear feeds, the only difference being the $90^{\circ}$ phase shift for Stokes $V$. 


\section{Zeeman splitting versus Chandrasekhar-Fermi}

A popular method for obtaining magnetic field strengths in atomic or molecular emission lines is the Chandrasekhar-Fermi $(\mathrm{CF})$ method. This relies on linear polarization and is sensitive to the plane-of-sky field $B_{\perp}$. The idea is this: the field is frozen into the gas; turbulence in the gas produces a dispersion in the field orientation, and hence the orientation of linear polarization; stronger fields make the system stiffer, so the dispersion is smaller. The bottom line: the square of the plane-of-sky field $\left(B_{\perp}\right)$ is proportional to the variance of the measured position angles.

The CF method almost always gives stronger field strengths than Zeeman splitting. There are at least three reasons for this:

(a) Statistically, $B_{\perp}$ is stronger than $B_{\|}$because it is closer to the total field strength $B_{\text {tot }}$. In essence, it is more probable for the field to lie in the plane of the sky than along the line of sight. Figure 6 shows this quantitatively by plotting the probability density functions (PDFs) and cumulative distribution functions (CDFs) for various ratios of field components. The CDFs are the most instructive: for half of all measurements, $B_{\|} / B_{\perp}<0.58$. Moreover, half the time $B_{\perp} / B_{\text {tot }}>0.87$, which makes $B_{\perp}$ a reasonably good tracer of $B_{\text {tot }}$.
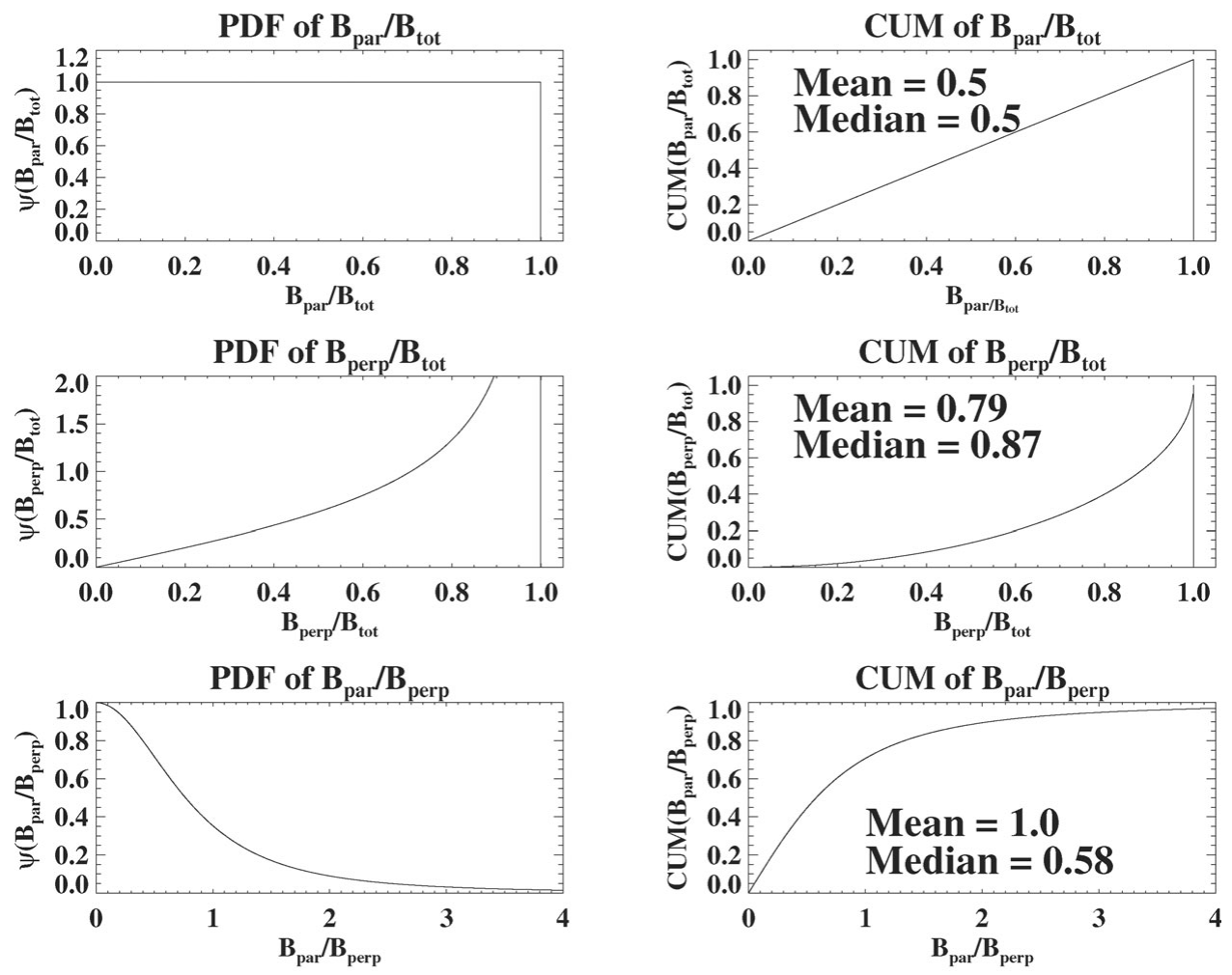

$\mathrm{X}$

Figure 6. The left column of panels exhibits plots of the probability density functions (PDFs) of $B_{\|} / B_{\text {tot }}, B_{\perp} / B_{\text {tot }}$, and $B_{\|} / B_{\perp}$. The right column exhibits the cumulative distribution functions of the same quantities.

(b) The CF method assumes that there is only a single polarizing cloud along the line of sight. This doesn't seem likely. With $N$ clouds and random field orientations among 
clouds, the derived field $\propto N^{1 / 2}$. Theorists have considered the question of how many elements should typically exist (e.g. Wiebe \& Watson 2004) and conclude that the effect is not too serious, but these predictions rely on turbulence models and theory. How much can we trust them?

(c) The CF method assumes that velocity fields are turbulent and random. However, interstellar space is subject to strong shocks from sources such as supernovae. These shocks will sweep up the field, remove the kinks, and make it look very smooth. An incredible example is the McClure-Griffiths et al. (2007) high-resolution HI map of the Riegel-Crutcher cloud, which shows a beautiful set of aligned angel-hair-like filaments; their Figure 6 shows that these filaments are aligned with stellar polarization. The CF method gives $B_{\perp} \approx 60 \mu \mathrm{G}$. This is huge and could easily be detected with Zeeman splitting. If so, it would be the strongest field detected in HI emission. It's worth a try!

\section{Acknowledgements}

This work was supported in part by NSF grant AST-0406987 and, also, by the awards GSSP 02-0011, 05-0001, 05-0003, 05-0004, and 06-0003 from the NRAO. It is a pleasure to acknowledge support from the IAU for this invited review.

\section{References}

Goodman, A. A. \& Heiles, C. 1994, ApJ 424, 208

Heiles, C. 1988, ApJ 324, 321

Heiles, C. 1989, ApJ 336, 808

Heiles, C. 1996, ApJ 466, 224

Heiles, C. 1997, ApJS 111, 245

Heiles, C. 1998, Astrophysics Letters and Communications, 37, 85. The title of this paper, "Zeeman Splitting Opportunities and Techniques at Arecibo", gives no hint that it discusses Veerschuur's Indictment. Moreover, this article is difficult to find on the internet. Copies are available at the authors' websites, currently http:astro.berkeley.edu/heiles and http://www.physics.usyd.edu.au/r̃obishaw)

Heiles, C. 1999, Arecibo Tech Memo 99-02 (http://www.naic.edu/science/techmemos_set.htm)

Heiles, C. 2002, in Single-Dish Radio Astronomy: Techniques and Applications, ASP Conf. Proceedings, Vol. 278. Eds S. Stanimirovic, D. Altschuler, P. Goldsmith, and C. Salter, 131

Heiles, C. \& Crutcher, R. 2005, LNP Vol. 664: Cosmic Magnetic Fields 664, 137

Heiles, C., Perillat, P., Nolan, M., Lorimer, D., Bhat, R., Ghosh, T., Lewis, M., O'Neil, K., Salter, C., \& Stanimirovic, S. 2001, PASP 113, 1274

Heiles, C. \& Troland, T. H. 2005, ApJ 624, 773

Lane, W. \& Heiles, C. 2008, in preparation

McClure-Griffiths, N. M., Dickey, J. M., Gaensler, B. M., Green, A. J., \& Haverkorn, M. 2006, ApJ 652, 1339

Myers, P. C., Goodman, A. A., Güsten, R., \& Heiles, C. 1995, ApJ 442, 177

Robishaw, T. \& Heiles, C. 2009, PASP, in press

Robishaw, T., Quataert, E., \& Heiles, C. 2008, ApJ 680, 981

Troland, T. H. \& Heiles, C. 1982, ApJ 252, 179

Turnshek, D. A., Rao, S., Nestor, D., Lane, W., Monier, E., Bergeron, J., \& Smette, A. 2001, ApJ 553, 288

Verschuur, G. L. 1969, ApJ 156, 861

Verschuur, G. L. 1989 ApJ 339, 163

Verschuur, G. L. 1995a, ApJ 451, 624

Verschuur, G. L. 1995b, ApJ 451, 645

Wiebe, D. S. \& Watson, W. D. 2004, ApJ 615, 300

Wolfe, A., Broderick, J., Condon, J., \& Johnston, K. 1976, ApJ 208, L47

Wolfe, A., Jorgenson, R., Robishaw, T., Heiles, C., \& Prochaska, J. 2008, Nature 455, 638

Wolleben, M. \& Reich, W. 2004, A\&A 427, 537 


\section{Discussion}

ZINNECKER: I was intrigued by your result that the measured magnetic fields are stronger in shock-compressed regions. Does this imply that the mass-to-flux ratio also changes upon shock-compression with consequences for star formation?

HeILES: Usually not; because during the shock compression which is one-dimensional, $\mathbf{B} \propto n$.

BECK: The detection of a $\approx 80 \mu \mathrm{G}$ field in a $z \approx 0.6$ galaxy is amazing, especially in view of the absence of any Faraday rotation. What is your physical picture of this object?

HeILES: It is very hard to understand. VLBI shows it is at least 200pc big, yet it has a narrow line with. This, by itself, is a puzzle, adding the magnetic pressure - 1000 times higher than thermal - makes it even harder. For a physical model we might think of two extremes: 1) The HI cloud is gravitationally confined. This needs a large surface density of stars (which would imply lots of star formation; not observed though) or Dark Matter. 2) An unconfined slab that was produced by a collision between two clouds, each having a moderate field strength of a few $\mu \mathrm{G}$, the shock waves compress the gas and the field; what is the correct magnitude? We have to find out with more data.

LAI: Can you talk about the first two problems of the CF method?

HeIles: $\mathrm{CF}$ is sensitive to $\mathbf{B}_{\perp}$, Zeeman is sensitive to $\mathbf{B}_{\|} \cdot 1$ ) The PDF (probability density function) is such that a) half the time, $\mathbf{B}_{\perp} / \mathbf{B}_{\text {total }}>0.87$, so $\mathbf{B}_{\perp}$ is a good tracer of $\left.\mathbf{B}_{\text {total }}, \mathrm{b}\right)$ half the time, $\mathbf{B}_{\|} / \mathbf{B}_{\text {total }}>0.5$, these two together give, c), half the time $\mathbf{B}_{\|} / \mathbf{B}_{\perp}<0.58$. 2. Stellar polarization averages elements over the line of sight. With $N$ randomly magnetic fields over many lines of sight the variance in position angles is reduced by a factor $N$. This increases the derived field by a factor $N$. 


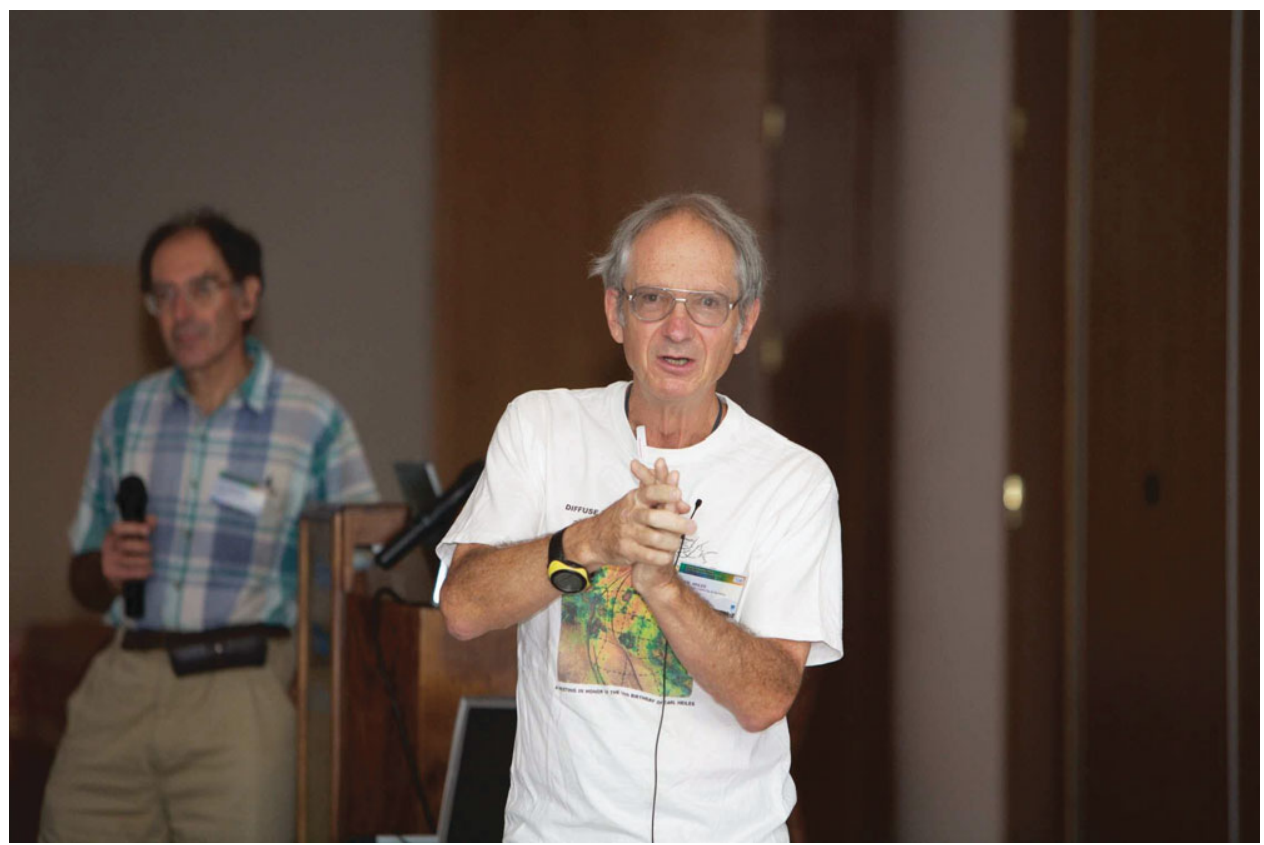

Carl Heiles, with the session chair Tomm Landecker in the background

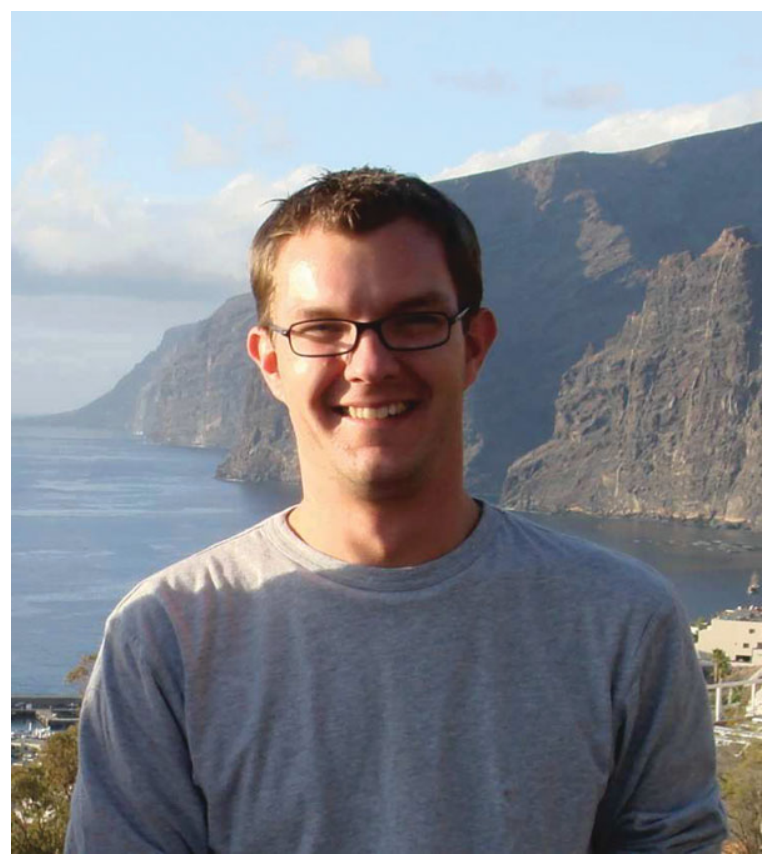

George Heald 